\title{
Multi-lead Fusion Detection of T-Wave Alternans Using Dezert-Smarandache Theory
}

\author{
Changrong Ye ${ }^{1}$, Guojun $\mathrm{Li}^{2}$, Yang Bao ${ }^{2}$, Jinliang Qiao ${ }^{2}$, Deping Zhang ${ }^{2}$ \\ 1) Chongqing University, Chongqing 400044; 2) Chongqing University of Posts and Telecommunications, 400065
}

\begin{abstract}
T-Wave alternans (TWA) is a cardiac phenomenon regarded as an index of high risk of sudden cardiac death (SCD). Although a number of methods have been proposed for TWA detection, their final decision always fully depends on one single lead which is usually picked out for its strongest TWA detection result among all the other leads. That is to say that lots of useful information have been unused and wasted. To our best knowledge, no method that fuses TWA detection results independently obtained by each lead to do comprehensive decision has been introduced. In this paper, a novel multi-lead method for TWA fusion detection is proposed. This method combines Dezert-Smarandache theory (DSmT) with Laplacian likelihood ratio method (LLR) to gain higher detection rate. The proposed method was evaluated and compared with standard LLR method by means of a simulation study, in which extracted TWA waveform and clean background ECG from real records were used to synthetize simulated data with different types of simulated or physiological noises. These methods were also applied to real records from PTB Diagnostic ECG Database. The results are presented by constructing receiver-operator characteristic (ROC) curves. All test results show that the proposed method has a larger margin of separability and higher detection rate than traditional methods. A more accurate and robust TWA detection method has great value to predict the risk of sudden cardiac death(SCD).
\end{abstract}

Index Terms-Dezert-Smarandache theory (DSmT), ECG, Laplacian likelihood ratio method (LLR), multilead fusion detection, T-wave alternans (TWA)

\section{INTRODUCTION}

$\mathrm{T}$ -WAVE alternans (TWA), also known as repolarization alternans, is a cardiac phenomenon, which is strongly associated with sudden cardiac death (SCD) [1], [2]. Visible TWA [see Fig. 1(a)] was first reported in 1908 by Hering ${ }^{[3]}$ and at that time it was believed to be rare for the lack of detection methodology. In 1981, Adam et al. reported the existence of the microvolt level TWA, which is difficult to detect for its small amplitude and the strong background noise ${ }^{[4]}$. Follow-up studies and clinical trials had demonstrated the existence of a link between TWA and SCD ${ }^{[5]}$, [6]. So an urgent need for accurate and robust TWA detection method arises from the demands to predict the risk for SCD.

TWA is defined as a beat-to-beat fluctuation in the amplitude, waveform, or duration of the ST-segment or T-wave [see Fig.

This work was supported in part by the National Natural Science Foundation of China under Grant 61671452 and National Natural Science Foundation of Chongqing (CSTC2015jcyjBX0078, CSTC2016jcyjA0556, ).

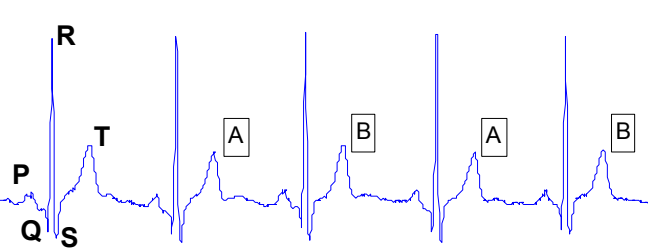

(a)

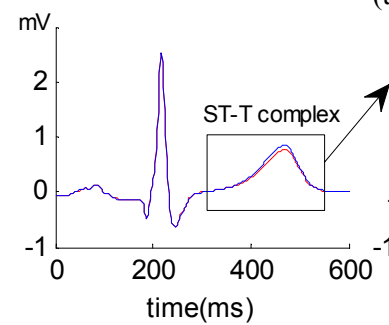

(b)

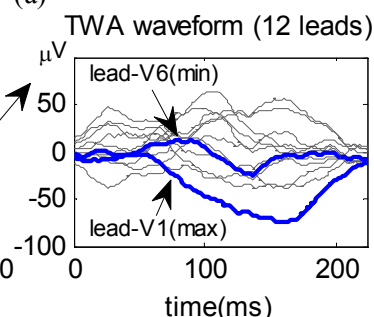

(c)
Fig. 1. (a) Single lead ECG signal with visible TWA. (b) Superposition of averaging waveform of odd and even beats. (c) Alternans waveform from different leads.

1(b)] and a lot of methods have been proposed for TWA analysis. Some of the most widely used single-lead TWA detection methods are the spectral method (SM) ${ }^{[7], ~}{ }^{[8]}$, the modified moving average (MMA) method ${ }^{[9]}$, the complex demodulation (CD) method [10] and Laplacian likelihood ratio (LLR) method ${ }^{[11],[12]}$. Based on these methods, some modified schemes were proposed ${ }^{[13]-[15]}$. A unified framework that holds these different approaches to TWA analysis can be found in [16], which represents the state of art for the existing single-lead TWA detection algorithms proposed in the literature.

However, due to the individual varieties of subjects and uncertainty of electrode placement in clinical environment, the potential TWA component may arise in any of several (or single) dimension of vector cardiogram (VCG), which lead to a very high false dismissal probability (also called false negative rate) for single-lead detection result. So when multi-lead ECG data available, a basic multi-lead strategy adopted by most of these single-lead TWA detection methods is that if TWA is detected at least in one lead then the overall TWA detection test is positive after the single-lead method is applied to each lead individually. (For short, the single-lead LLR method with this strategy is called "OR" method in following sections.) Some multi-lead schemes for TWA detection and analysis have also been proposed, as described in [17], [18], which take advantage of the correlation of background ECG between leads to de-noise and reconstruct the multi-lead ECG signal by using principal component analysis (PCA). However, for the decision 
part, the same 'OR' strategy as most single-lead methods adopted is performed.

One of the main drawbacks in all these existing multi-lead TWA detection methods mentioned above is that only TWA component from one lead is used to do the final decision, which leads to low utilization rate of data and high sensitivity to impulse noise. In some cases, the 'OR' strategy may even worsen the detection performance when compared to the result obtained from specific lead. But multi-lead fusion TWA detection by data from all leads is a difficult task for reasons below. First, the differences of morphology and amplitude of TWA waveform in each lead [see Fig. 1(c)] make it difficult to utilize the correlation between leads to extract a fusion TWA waveform by multi-lead signal processing methodologies such as PCA. Second, the high conflict between detection results from different leads and the different definitions between single-lead hypotheses and overall hypotheses make it improper to apply existing fusion methodologies directly. That is to say, a test subject may be regarded as positive for TWA detection test if TWA is detected in certain lead, but it cannot be regarded as negative if TWA is not detected in this lead. To our best knowledge, no study to address these issues has been introduced so far.

In this paper, a multi-lead fusion TWA detection methodology that combines Dezert-Smarandache Theory (DSmT) with LLR method is proposed. The proposed fusion detection framework based on DSmT can work with different single-lead methods after a few modifications. LLR is chosen here for its robustness to noise. A detailed comparison of performance between LLR and other traditional single-lead methods just like SM and MMA can be found in [11], [12]. DSmT of plausible and paradoxical reasoning is introduced to fusion results from leads into an overall detection result. The result obtained by single-lead method from single-lead data cannot be used as input of DSmT fusion processing directly, so some transformations are used to transform these results into basic belief assignment (bba) which can be regarded as the universal format of input or output of DSmT fusion processing. Besides, in order to evaluate the performance of proposed method theoretically, synthetic ECG records with high degree of realism were created and a Monte Carlo simulation approach was adopted. Experiments on real ECG datasets from public databases were also conducted to evaluate its clinical performance.

The remainder of the paper is organized as follows. Section II introduces the LLR method for single lead TWA detection and DSmT for fusion decision, followed by a detailed description of proposed TWA fusion detection method. Section III is presented with detail of datasets and relevant experiments. Results are shown in Section IV. Finally, the discussion and conclusions are given in Section V and VI respectively.

\section{METHODS}

\section{A. LLR Method}

The LLR method was firstly proposed in [11], [12]. Under assumption of Laplacian noise, this method adopts a generalized likelihood ratio test (GLRT) on single-lead data to decide whether TWA is present or not.

Just like the most other single-lead methods for TWA detection, in the first place, consecutive ST-T complexes are extracted, aligned and put into an ST-T matrix which can be expressed as

$$
\mathbf{X}=\left[\begin{array}{lll}
\mathbf{x}_{1} & \cdots & \mathbf{x}_{K}
\end{array}\right]=\left[\begin{array}{ccc}
x_{1}(0) & \cdots & x_{K}(0) \\
\vdots & \ddots & \vdots \\
x_{1}(N-1) & \cdots & x_{K}(N-1)
\end{array}\right]
$$

where $N$ is the number of samples in each ST-T complex and $K$ is the number of beats under analysis. In LLR method, the ST-T complex of $k^{\text {th }}$ beat is modelled as

$$
\mathbf{x}_{k}=\mathbf{s}+\mathbf{a}(-1)^{k}+\mathbf{v}_{k}
$$

where $\mathbf{S}$ is the background ST-T complex vector, $\mathbf{a}$ is the TWA waveform and $\mathbf{v}_{k}$ is the vector of additive noise in $k^{\text {th }}$ beat. Since the noise is assumed zero-mean and $K$ is even, the background ST-T complex can be canceled easily by

$$
\mathbf{x}_{k}^{\prime}=\mathbf{x}_{k}-\frac{1}{K} \sum_{k=1}^{K} \mathbf{x}_{k}=\mathbf{a}(-1)^{k}+\mathbf{v}_{k}, \quad k=1, \ldots, K .
$$

Since random process $\mathbf{v}_{k}$ is assumed zero-mean and its probability distribution function (pdf) is symmetrical, $\mathbf{x}_{k}^{\prime}$ can be simplified by

$$
\mathbf{y}_{k}=\mathbf{x}_{k}^{\prime}(-1)^{k}=\mathbf{a}+\mathbf{v}_{k}, \quad k=1, \ldots, K .
$$

The maximum likelihood estimation (MLE) of $\mathbf{a}$ is given by Table-I in [12]

$$
\hat{\mathrm{a}}(n)=\operatorname{median}\left(\left\{y_{k}(n)\right\}_{k=1}^{K}\right), \quad n=0, \ldots, N-1 .
$$

With the hypothesis testing problem

$$
\begin{aligned}
& \mathrm{H}_{0}: \mathbf{a}=\mathbf{0} \\
& \mathrm{H}_{1}: \mathbf{a} \neq \mathbf{0},
\end{aligned}
$$

the GLRT statistic can be expressed as

$$
Z=\frac{\sum_{n=0}^{N-1} \sum_{k=1}^{K}\left|y_{k}(n)\right|}{\sum_{n=0}^{N-1} \sum_{k=1}^{K}\left|y_{k}(n)-\hat{\mathrm{a}}(n)\right|} .
$$

Then the GLRT result $Z$ is compared with a threshold $\lambda$, if $Z \geq \lambda$ the detection results is positive, otherwise negative.

Before going further, it is worth to note that the output result $Z$ essentially represents the ratio between TWA component and average noise [12]. Based on this idea, GLRT result $Z$ can be expressed as

$$
Z=\sqrt{\frac{E_{\text {noise }}+E_{\text {twa }}}{E_{\text {noise }}}}
$$

where $E_{\text {noise }}$ and $E_{\text {twa }}$ are the estimations of energy of average noise and TWA component during ST-T segment respectively. Different single-lead methods have their own ways to estimate the amplitude or energy of TWA component 
and noise, but most of them finally result in similar a ratio just like $Z$ in LLR. That's why the proposed DSmT fusion method may be able to compatible with other similar single-lead method with a few modifications.

\section{B. Dezert-Smarandache Theory}

Evidence theories are widely applied in the field of information fusion and DSmT is one of the most appealing and promising theories. The task of DSmT is to combine imprecise, uncertain, incomplete, qualitative or quantitative and possibly conflicting information under consideration for decision-making support. A short introduction to DSmT can be found in [19]. More details and examples can be found in [20]. DSmT is extended and refined from Dempster-Shafer Theory (DST) [21] by proposing new underlying models for the frames of discernment (FOD) in order to fit better with the nature of real problems, and new combination and conditioning rules for circumventing problems with DS rule especially when the sources to combine are highly conflicting. More details about the relationship between DST and DSmT can be found in [22]. Three basic parts of DSmT that will be used in this paper are briefly recalled as follows:

- Frame of Discernment and Basic Belief Assignment: In DSmT framework, all the sources of evidences must be unified and transformed into (generalized) basic belief assignment (bba also called basic probability function or mass function) ${ }^{[20]}$ based on the same frame of discernment (FOD). FOD of the problem under consideration is defined as a finite set $\Theta=\left\{\theta_{1}, \theta_{2}, \ldots, \theta_{n}\right\}$ which consists of all possible hypotheses $\theta_{i}, i=1, \ldots, n$. In order to deal with vague/fuzzy and relative concepts, all the hypotheses (or elements) are not necessarily exclusive and there is no restriction except exhaustivity. To describe all propositions built from hypotheses of $\Theta$ with $U$ and $\cap$, a fusion space called hyper-power set $D^{\ominus}$ (Dedekind's lattice) is introduced and the mapping function $m():. D^{\Theta} \rightarrow[0,1]$ is defined as bba which satisfies:

$$
\sum_{A \in D^{\Theta}} m(A)=1 \text { and } m(\varnothing)=0 \text {. }
$$

In this paper, in order to be compatible with single-lead method, the hypotheses are defined the same as (6) and the FOD can be expressed as

$\Theta=\left\{\theta_{1}=H_{1}=\right.$ overall positive, $\theta_{2}=H_{0}=$ overall negative $\}$.

Since the fact that the result of GLRT cannot be recognized as positive and negative at the same time, the two hypotheses are considered truly exhaustive. So the hyper-power set $D^{\Theta}$ is simplified as a power set $2^{\ominus}$ which can be expressed as

$$
D^{\Theta}=2^{\Theta}=\left\{A=\theta_{1}, B=\theta_{2}, C=\theta_{1} \cup \theta_{2}\right\} .
$$

That is to say, before fusion process, GLRT result from $n^{\text {th }}$ lead must be transformed into a bba on power set $2{ }^{\Theta}$ which can be expressed as a vector

$$
\mathbf{m}_{n}=\left[m_{n}(A) m_{n}(B) m_{n}(C)\right]^{\mathrm{T}} .
$$

DSmT, many rules were proposed to combine basic belief assignments (bba's) and Proportional Conflict Redistribution no. 5 (PCR5) is one of the most efficient rules. The basic idea of PCR5 is transferring the conflicting mass only to the elements involved in the conflict and proportionally to their individual masses to preserve all input information in the fusion process. A variant of PCR5, called Proportional Conflict Redistribution no. 6 (PCR6), for combining $s>2$ sources and working in more generalized fusion spaces (hyper-power sets or super power-sets), was firstly proposed by Martin and Osswald in [20], Vol. 2. PCR6 can be expressed as

$$
\begin{aligned}
& m_{P C R 6}(X)=m_{12 \ldots . .}(X)+ \\
& \sum_{i=1}^{s} m_{i}(X)^{2} \sum_{\bigcap_{i=1}^{s-1} Y_{\sigma_{i}(k)} \cap X \equiv \varnothing}\left[\frac{\sum_{j=1}^{s-1} m_{\sigma_{i}(j)}\left(Y_{\sigma_{i}(j)}\right)}{m_{i}(X)+\sum_{j=1}^{s-1} m_{\sigma_{i}(j)}\left(Y_{\sigma_{i}(j)}\right)}\right] \text { (14) } \\
& \left(Y_{\sigma_{i}(1)}, \ldots, Y_{\sigma_{i}(s-1)}\right) \in\left(2^{\theta_{g}}\right)^{s-1} \\
& m_{12 \ldots s}(X)=m_{1}(X) m_{2}(X) \ldots m_{s}(X) \\
& \sigma_{i}=\left\{\begin{array}{cc}
j, & j<i \\
j+1, & j \geq i
\end{array}\right. \text {. }
\end{aligned}
$$

More details about PCR5/PCR6 can be found in [20].

Since the number of leads that may access in clinical setting is usually larger than 2 (3 for Frank XYZ leads and 12 for conventional leads), PCR6 will be used in this paper to combine bba's built on fusion space defined in (11).

- Decision-making: A final decision based on bba must be taken after fusion process. Various decision rules are proposed in the literature. The most common are the maximum credibility and maximum plausibility and the probability. Based on these common rules, many variant decision rules, just like Generalized Pignistic Transformation (GPT), DSmP and Sudano's rule, etc. provide more reasonable options for specific situations. Consequently, the maximum credibility rule was chosen for its simplicity of implementation in this paper.

\section{Multi-lead Scheme}

The block diagram of the proposed multi-lead scheme is shown in Fig. 3. It consists of five stages: signal preprocessing, ST-T matrix generation, LLR detection, bba generation \& unification and fusion detection.

1) Signal Preprocessing: In signal preprocessing stage, the level of out-of-band noises in raw signal is reduced and ST-T complexes are extracted and aligned to construct a matrix of ST-T complex lead by lead. To achieve this goal, we utilized the similar preprocessing steps across all methods ${ }^{[24]}$. Firstly, in order to eliminate high-frequency noise, a low-pass-filter (LPF) with a cutoff frequency of $150 \mathrm{~Hz}$ is adopted. Secondly, baseline wander is removed with a cubic splines interpolation technique. Thirdly, a QRS detector from PhysioToolkit ${ }^{[25]}$, whose algorithm can be found in [26], [27], is used to locate the fiducial point and then the position of each ST-T complex. Fourthly, 32 (which required by LLR method) consecutive ST-T complexes from a single lead are extracted to construct 


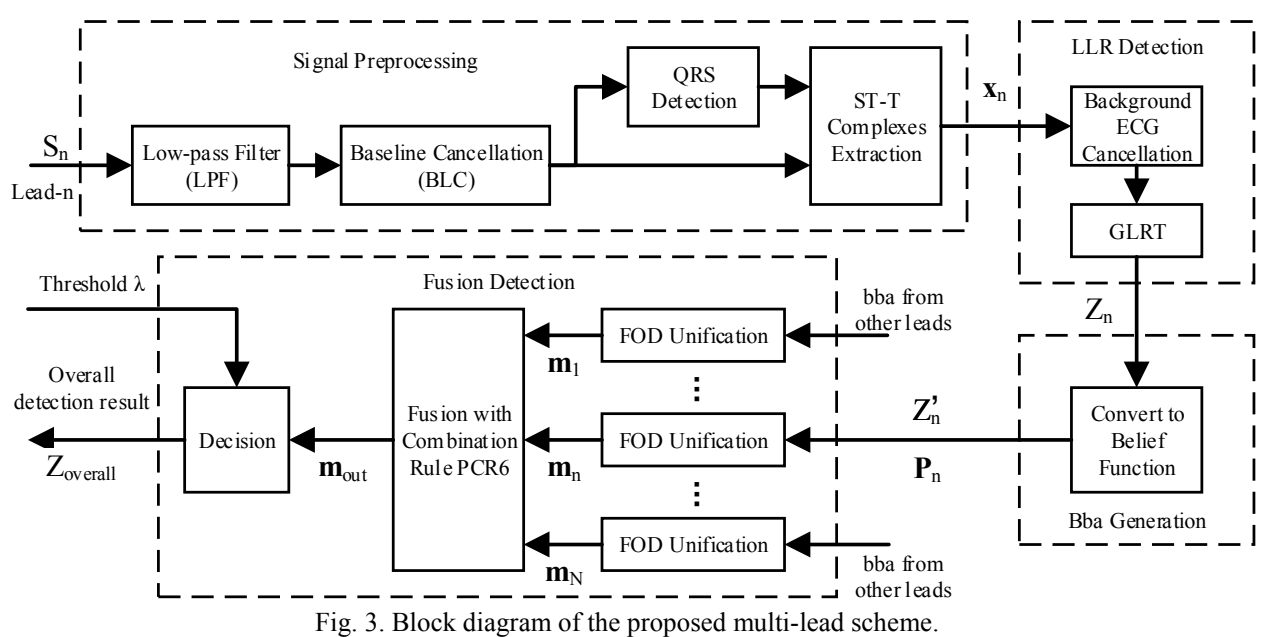

the ST-T matrix $\mathbf{X}_{n}$ of $n^{\text {th }}$ lead.

2) LLR Detection: After signal preprocessing, a standard single-lead LLR method is performed in the matrixes of ST-T complex to do TWA detection. The background ECG are firstly removed by (3) and then the GLRT is applied to each matrix of ST-T complex as shown in Section II-A. The GLRT result of this lead-by-lead detection is denoted as $Z_{n}$. In traditional strategy, the overall TWA detection is positive if TWA is detected at least in one matrix of ST-T complex. In proposed multi-lead scheme, the GLRT results $Z_{n}, n=1,2, \ldots, N$ are delivered to next stage to do fusion detection as shown in Fig. 3.

3) Bba Generation: In bba generation stage, the GLRT results are transformed into bba's. According to the description of (8), $Z_{n}^{\prime}$ is designed as the ratio of power of TWA to noise estimation as

$$
Z_{n}^{\prime}=\frac{E_{t w a}}{E_{\text {noise }}} .
$$

Combining it with (8), the single-lead GLRT results are transformed into $Z_{n}^{\prime}$ by

$$
Z_{n}^{\prime}=Z_{n}^{2}-1,
$$

which can be expressed as bba $m_{n}$ of $n^{\text {th }}$ lead

$$
\begin{aligned}
& m_{n}\left(A_{s}\right)=Z_{n}^{\prime} \\
& m_{n}\left(B_{s}\right)=1-Z_{n}^{\prime}
\end{aligned}
$$

where single-lead FOD is defined as

$$
\Theta_{s}=\left\{A_{s}=\text { single lead positive, } B_{s}=\text { single lead negative }\right\} \text {. }
$$

4) Fusion Detection: In fusion detection stage, PCR6 is applied to combine all these bba's from each lead into one. It is worthy to note that, for TWA fusion detection, the definitions of hypothesizes in (10) are not uniformly the same as hypothesizes in (22). To unify the definition of FOD before PCR6 fusion, a step called FOD unification is performed by

$$
\begin{aligned}
& m_{n}(A)=P_{n}\left(A_{s}\right) \\
& m_{n}(B)=\prod_{\mathrm{n}=1}^{N} P_{n}\left(B_{s}\right) \\
& m_{n}(C)=1-P_{n}\left(A_{s}\right)-P_{n}\left(B_{s}\right)
\end{aligned}
$$

where hypotheses $\{A, B, C\}$ are defined in (11).

The basic idea here is that the overall detection result may be regarded as positive if TWA is detected in at least one lead (i.e. $A=A_{s}$ ), but the overall result cannot be regarded as positive if TWA is not detected in a specific single lead (i.e. $B \neq B_{s}$ ). The spare mass of $B_{s}$ is redistributed to $C$ which represents the uncertain mass that will be determined by other leads. Then, the fusion based on PCR6 is performed as described in Section II-B.

After fusion process, maximum credibility rule is used to do final decision based on $m_{\text {overall }}$. For ease of comparison between single-lead method and multi-lead scheme, $Z_{\text {overall }}$ is defined as

$$
Z_{\text {overall }}=m_{\text {overall }}(A)
$$

which means that if $Z_{\text {overal }} \geq \lambda$, the overall TWA detection overall result is positive, otherwise negative.

\section{EXPERIMENTS}

In this section, records from three two databases are used to evaluate the proposed multi-lead fusion detection method. How these signals synthesized or recorded is described and then three methods (LLR method on single-lead data, LLR method on multi-lead data with "OR" strategy and proposed multi-lead method) are applied to detect TWA component on these records. The results are shown and the performances of the three methods are compared.

\section{A. Synthetic Signal Database}

To evaluate the improvement of proposed multi-lead scheme theoretically, a Monte Carlo simulation study was carried out.

Since the actual presence of alternans is unknown in real records, a synthetic database was generated and the process of synthesizing ECG records with TWA is shown in Fig. 4. 


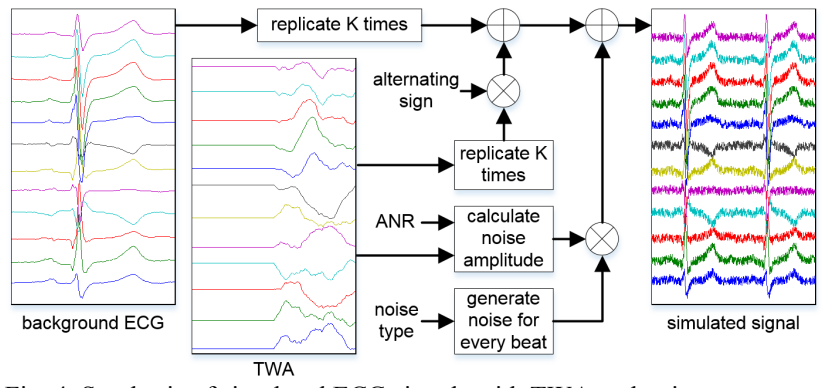

Fig. 4. Synthesis of simulated ECG signals with TWA and noise.

Synthetic records were generated by adding noise and TWA to a clean background ECG. The synthesis scheme was designed on the basis of simulation setup in [18]. Four types of noise were added, and they were Gaussian (ga) noise, Laplacian (la) noise, electrode motion (em) noise and muscular activity (ma) noise. Noise 'ga' and 'la' were generated randomly and 'em' and 'ma' were extracted from real noise records from MIT-BIH Noise Stress Test Database. More details about these 2 types of noise and how these records were recorded can be found in [28]. Because each single-lead data are processed individually by all the methods to apply, simulation of correlation between different types of noise in different leads is not necessary in this simulation study. To synthesize multi-lead ECG records with TWA as universal as possible, the TWA waveform was extracted from 4 records: "s02601re", "s0261lre", "s0265lre" and "s0315lre" by standard LLR method lead by lead. All these
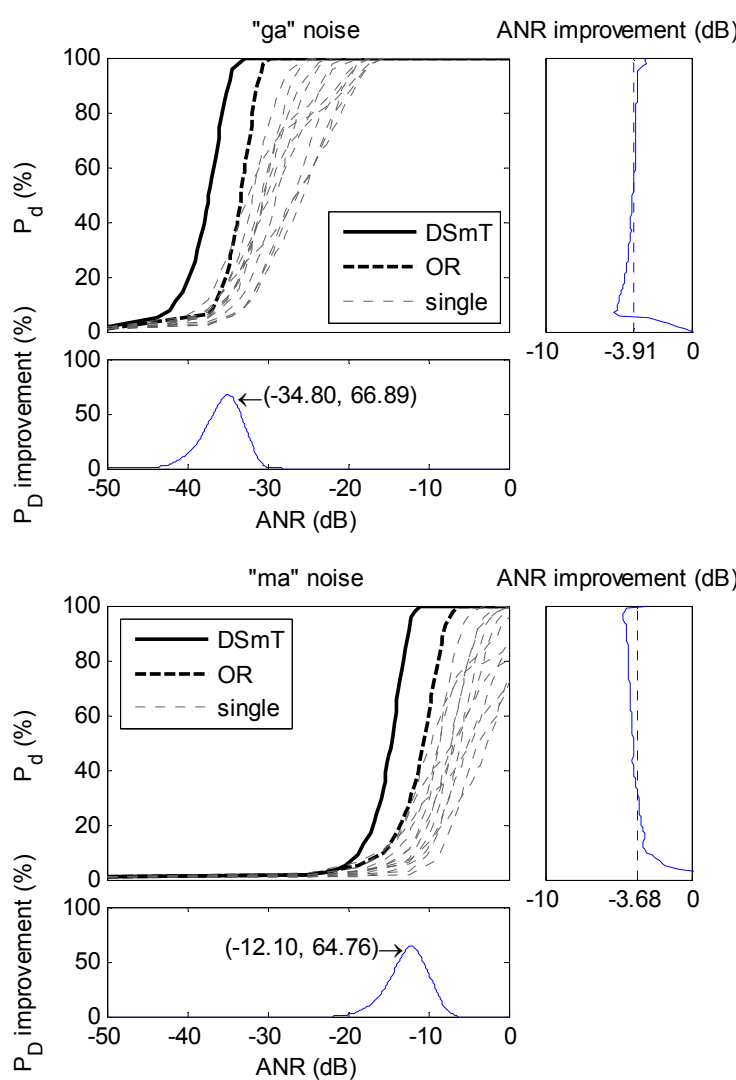

records come from "patient080" which have visible TWA. Alternans-to-noise (ANR) is widely used to access noise in TWA simulation study as known priori [11], [14], [29] and it is defined as the averaged relationship between the alternant wave power and the noise power in the ST-T complexes from one single lead. Considering multi-lead background, if the $\mathbf{a}_{k}$ and $\mathbf{v}_{k}$ of $l^{\text {th }}$ lead in (2) can be also expressed as $\mathbf{a}_{l, k}$ and $\mathbf{v}_{l, k}$ respectively, traditional definition of ANR of $l^{\text {th }}$ lead can be defined as

$$
A N R_{l}=10 \log \frac{\sum_{k=1}^{K} \sum_{n=0}^{N-1} \mathrm{a}_{l, k}(n)^{2}}{\sum_{k=1}^{K} \sum_{n=0}^{N-1} \mathrm{v}_{l, k}(n)^{2}} .
$$

In this paper, this definition is expanded as overall ANR to access noise in multi-lead signals, which can be defined as

$$
A N R_{\text {overall }}=10 \log \frac{\sum_{l=1}^{L} \sum_{k=1}^{K} \sum_{n=0}^{N-1} \mathrm{a}_{l, k}(n)^{2}}{\sum_{l=1}^{L} \sum_{k=1}^{K} \sum_{n=0}^{N-1} \mathrm{v}_{l, k}(n)^{2}} .
$$

All the ANR mentioned below refers to $\mathrm{ANR}_{\text {overall }}$ unless otherwise stated. Besides, it is worthy to note that, to eliminate the effects of ST-T complex location error, which should not be ignored when ANR is low, the locations of ST-T complexes were determined before noise was added. For each type of noise and each specific ANR, 1000 records were generated for
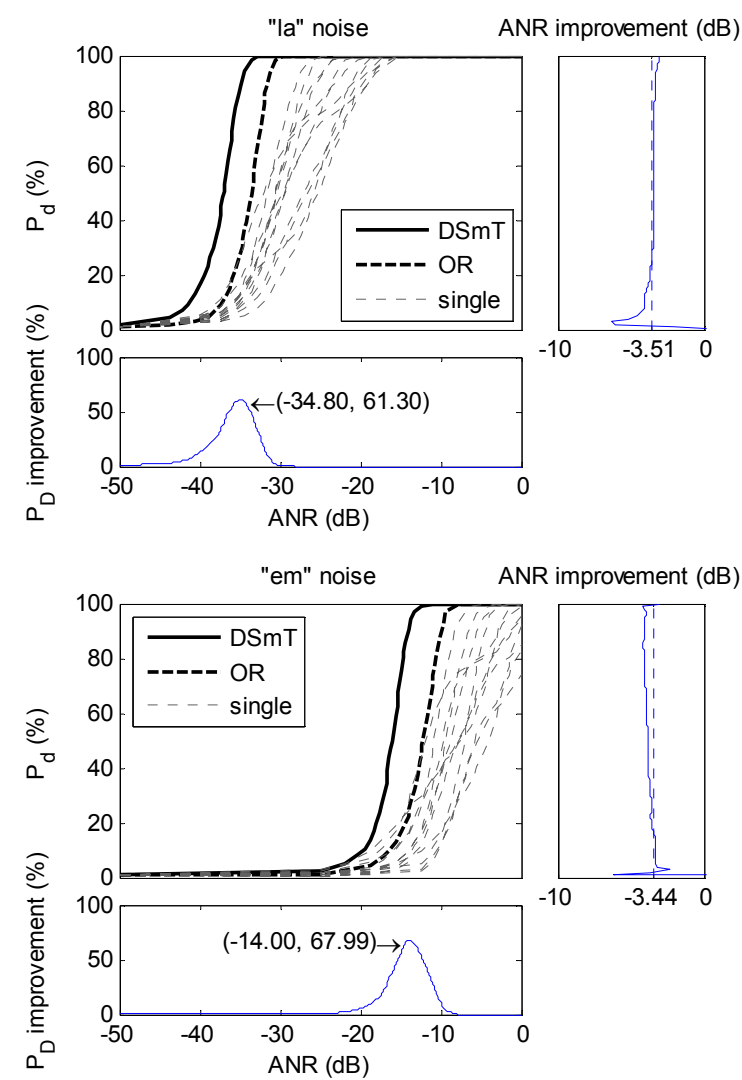

Fig. 5. $\mathrm{P}_{\mathrm{D}}$ for $\mathrm{P}_{\mathrm{FA}}=0.01$ of the proposed DSmT fusion method, traditional "OR" method and standard LLR method on each single lead versus ANR. All results obtained with an analysis window of 32 beats. Sub-figure below shows the difference between $P_{D}$ of proposed DSmT fusion method and traditional "OR" method with the same ANR. Sub-figure on the right side shows the difference of ANR with same PD of proposed DSmT fusion method and traditional "OR" method. The mean difference is shown in dotted line. 

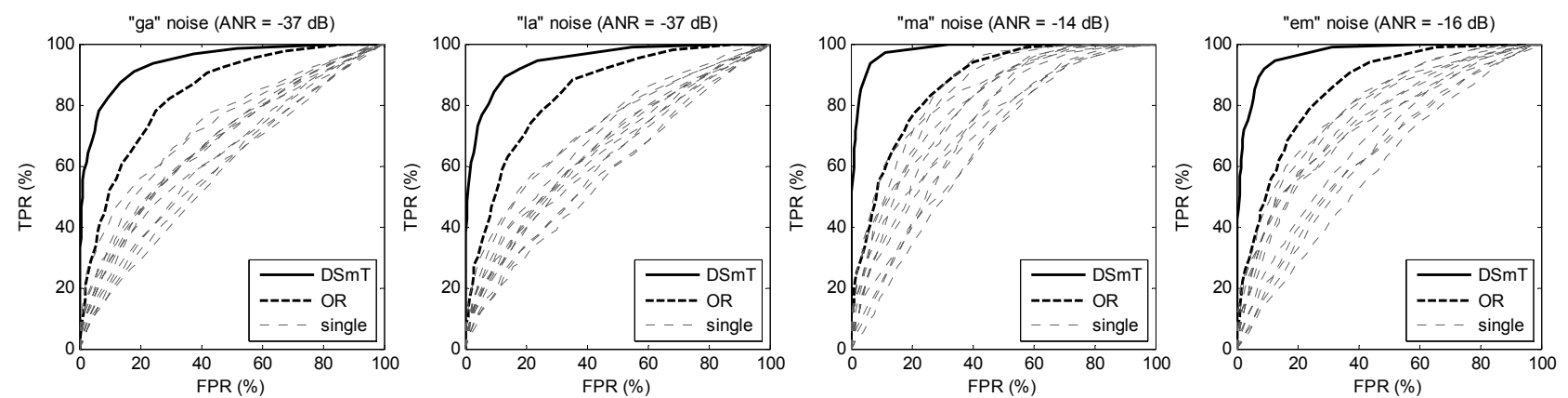

Fig. 6. ROC curves of detection results obtained by proposed DSmT fusion method, traditional "OR" method and standard LLR method on each single lead.

the Moto Carlo simulation. The detection rate of each 1000 synthetic records is defined as the percentage of tests whose results are positive.

For each method to test, the experiment was carried out in 2 steps. Firstly, calculate the threshold, which made the detection rate on the 1000 synthetic records without TWA ( ANR $=-\infty$ ) equal to 0.01 . This rate is defined as probability of detection, which called False Positive Rate (FPR) in detection problem. And then with this threshold, the detection rates on synthetic records with TWA whose ANR ranges from $-50 \mathrm{~dB}$ to $0 \mathrm{~dB}$ were calculated. This rate is also called True Positive Rate (TPR) and the results are shown in Fig. 5. Secondly, with specific ANR, FPR and TPR with threshold ranges from 0 to 1 were calculated. The results are shown as ROC curves in Fig. 6.

In Fig. 5, TPR vs. ANR when FPR $=0.01$ are shown. The maximum improvement $67.99 \%$ can be observed when $\mathrm{ANR}=-14 d B$ with 'em' noise. Besides, the 'OR' method is not always better than single-lead method. In cases of ' $\mathrm{ga}$ ' and TABLE I

PERFORMANCES OF DETECTORS (S95)

\begin{tabular}{cccc}
\hline \hline & & SM & LLR \\
\hline \multirow{2}{*}{ S95(\%) } & OR & 6.76 & 20.27 \\
& DSmT & 14.86 & 29.05 \\
\hline
\end{tabular}

'ma' noise, some single-lead methods have better performance than 'OR' method when ANR is low. In Fig. 6, ROC curves are drawn to show the FPR and TPR as a function of the detection thresholds $\gamma$ with a fixed ANR. It is obvious that the area under the ROC curves of the DSmT fusion detection method was greater than the area under curves of "OR" strategy based on single-lead LLR method which means a better detection performance.

\section{B. PTB Diagnostic Database}

As an example of application to real data, the proposed DSmT method was applied to a set of records from PTB Diagnostic Database. This database was provided by National Metrology Institute of Germany, which is also known as Physikalisch-Technische Bundesanstalt (PTB). These records were collected by Professor Michael Oeff, M.D. from patients and healthy volunteers. Each record was digitalized at 1000 samples per second, with 16-bit resolution over a range of \pm $16.384 \mathrm{mV}$ with 15 leads which including 12 conventional leads and 3 Frank leads.
To acquire universal result with adequate signals, two groups of records labeled with "healthy control" and "myocardial infarction", which contain 368 records from 148 patients and 80 records from 52 healthy volunteers respectively, were used to be analyzed by single-lead LLR methods, "OR" method and proposed DSmT method. Considering computational complexity and practical clinical setting, only 12 conventional leads were used in this experiment. The results of tests on subjects of "healthy control" and "myocardial infarction" by different methods are shown as ROC curves in Fig. 7.

To show the compatibility with other single-lead method, similar tests which use standard SM method as the single-lead method rather than LLR method were applied on the same records. The single-lead SM detection result $Z_{s m}$, which can be expressed as

$$
Z_{s m}=\frac{p s d_{0.5}-E\left(p s d_{0.4 \sim 0.46}\right)}{E\left(p s d_{0.4 \sim 0.46}\right)}=\frac{E_{t w a}}{E_{n o i s e}}=Z_{n}^{\prime},
$$

is directly used to construct input bba $m_{n}$ of $n^{\text {th }}$ lead by (20). The $p s d_{0.5}$ represents the alternans power which is the amplitude of power spectral density (pdf) at 0.5 cycles per beat (cps). The $E\left(p s d_{0.4 \sim 0.46}\right)$ represents the power of background noise which is calculated as the mean amplitude of pdf during $0.4 \sim 0.46$ cps. Though SM method generally use a threshold $\lambda=3$ to make the single-lead decision, the threshold was not fixed during calculating ROC curves. The results are shown as ROC curves in Fig. 7.

It should be specially explained that the length of data for each method is 129 beats for every records. The standard SM method require 129 beats while LLR method require 33 beats for every time. So a sliding window with 16 beats for every step in time is adopted by LLR method and the maximum result will be picked out to be the final result of corresponding lead. When some records do not have 129 beats, all the data were used to do single-lead TWA detection.

From ROC curves in Fig. 7, one can draw a conclusion that whichever of SM and LLR method is chosen as the single-lead detection method, DSmT fusion detection generally achieve improvement of detection power, especially in FPR range below $10 \%$. Besides, to compare these ROC curves in a simple way, the parameter called S95 proposed in [12] was calculated and shown in Table I. 


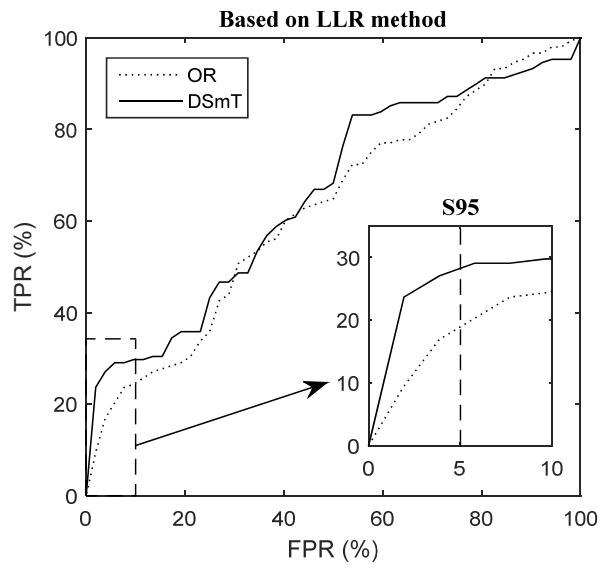

(a)

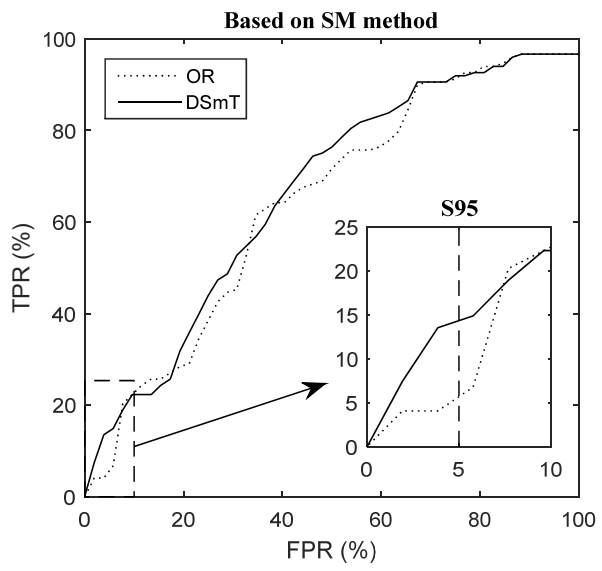

(b)

Fig. 7. Comparison between ROC curves of detection results on real records from PTBDB obtained by OR method and DSmT method based on different single-lead method: (a) LLR and (b) SM method.

\section{DISCUSSION}

According to simulation results, the proposed DSmT method has higher performance than "OR" method and single-lead LLR method. For Gaussian ("ga") noise, Laplacian ("la") noise, muscular activity (ma) and electrode motion (em) noise, the maximum improvements are $66.89 \%, 61.30 \%, 64.76 \%$ and $67.99 \%$. From the point of ANR reduction, the proposed DSmT method can achieve the same detection power with $3.91 \mathrm{~dB}$, $3.51 \mathrm{~dB}, 3.68 \mathrm{~dB}$ and $3.44 \mathrm{~dB}$ lower ANR for "ga","la","ma" and "em" noise respectively.

It is worthy to note that, in Fig. 5, the usually used "OR" method does not always have a better detection performance than all the single-lead methods especially when ANR is low. This is because that "OR" method improves both detection sensitivities of TWA and noise. With high ANR, the TWA becomes easy to detect by "OR" method while the noise has little effect. However, when ANR is low, noise suppresses TWA component and the FPR becomes high for the "OR" method, which is more likely to misdiagnose noise as TWA than single-lead method. To keep FPR fixed, threshold of "OR" method becomes lower than that of single-lead methods which naturally resulting in a reduction of TPR. Even so, the "OR" method is still widely used because in practical TWA detection, the distribution of TWA among leads and true ANR are unknown and varies from people to people, even time to time. So single-lead method with fixed lead cannot always ensure existence of TWA component with a high ANR, instead, it may cause a very high False Negative Rate (FNR). Following these analysis, a conclusion can be made that the "OR" method is a simple and crude fusion strategy which is adopted for reducing the FNR caused by the uncertain distribution of TWA among leads at the cost of low detection rate when ANR is low.

The proposed DSmT method not just works in simulation, but also on real records. The area under ROC curves obtained by DSmT fusion detection method is obviously larger than that by single-lead method including LLR and SM respectively in Fig. 7(a) and Fig. 7(b). Clinically, priority is usually given to ensure a low FPR, so the sections of ROC curves in FPR ranging below $10 \%$, which have been enlarged in Fig. 7, should be given more attentions.

It is worthy to note that the sections of ROC curves in FPR ranging above $20 \%$ do not show obvious superior to TPR obtained by "OR" method, and in a few sections, the TPR obtained by "OR" method is even larger than TPR obtain by DSmT method. For this part of test results, some major aspects will be discussed as follows.

First, the definitions of TPR of ROC curves in simulation study and tests on real records are different. In simulation study, TPR means proportion of the number of records detected as positive among all records which contains TWA component for sure. But in tests on real records, TPR means proportion of the number of records detected as positive among all records from patients which may not show any TWA during recording.

Second, concluded from simulation results shown in Fig. 5, the improvement gained by DSmT method is mainly concentrated in certain range of ANR with any types of noise. Detection results obtained by no mater "OR" or DSmT method on records with too high or too low ANR are similar. In tests on real records, the true ANR of real records are unknown. So just part of patients' records will be identified as positive while they are missed by single-lead method.

Third, the traditional TWA detection is usually carried out on stress test records which have a higher percentage of records containing TWA component among all patients' records. Stress test records are usually related to strenuous exercise which is not appropriate for quite a part of patients. This recording method also counts against long-term TWA monitoring for high risky populations in daily life. So in this study, conventional long-term (Holter) ECG recordings from PTB Diagnostic Database are used to observe TWA in the context of activities of daily living. This may lead to a relatively poor performance in general, but with relatively low ANR, the advantages of DSmT in fusing and dealing with conflicting information are fully shown.

Besides, the proposed DSmT method is far from perfect and it also has some problems to solve and there is still room for improvement. First of all, like many other methods based on DSmT, the proposed multi-lead method is restricted by the 
large computational complexity. The amount of computations increases sharply as that of leads and hypotheses in $2^{\Theta}$ increase. Let $L$ be the number of leads and $K$ be the number of hypotheses in $2^{\Theta}$, the number of masses on fusion space will be $n_{m}=K^{L}$. In the simulation of this paper, $K=3, L=12$ and $n_{m}=3^{12}=531441$,which will spend about $13.51 \mathrm{~s}$ with Intel E8400@3.6GHz for each DSmT fusion detection. Besides, the improvement of proposed DSmT method is significantly related to the number of leads, and the more leads take part in the fusion detection, the more information can be made use of in detection, the better performance of proposed method will have. When the leads lack in numbers, the improvement will be very limited. The most promising potential improvement may lie in estimating the importance of each lead and using this information in DSmT fusion process to gain more accurate detection results. Many factors (just like ANR, power of ST-T wave and residual of baseline) may have impact on the credibility and accuracy of detection results of each lead. How to use single factor or combine some of them to estimate the weights of each lead will be the focus of future researches.

\section{CONCLUSION}

In this paper, a novel multi-lead TWA fusion detection method in ECG was proposed. The proposed DSmT method was designed based on LLR method and DSmT. By combining these two methods, the proposed method can make full use of all information from every lead to gain a higher detection rate than standard single-lead LLR method and its variant called "OR" method in TWA detection. The proposed method was firstly assessed by a set of synthetic records with different types of noise and specific ANR as priori, then it was applied to a set of real records. All results from these experiments showed improvements in detection rates. Finally, we analyzed the experiment results and discussed its advantages, disadvantages and what should be done in the future to further enhance its detection power.

\section{REFERENCES}

[1] S. M. Narayan, "T-wave alternans and the susceptibility to ventricular arrhythmias," J. Amer. Coll. Cardiol., vol. 47, no. 2, pp. 269-281, 2006.

[2] V. Monasterio, P. Laguna, I. Cygankiewicz, R. Vázquez, A. Bayés-Genís, A. Bayés de Luna, and J. P. Martínez, "Average T-wave alternans activity in ambulatory ECG records predicts sudden cardiac death in patients with chronic heart failure," Heart Rhythm, vol. 9, no. 3, pp. 383-389, 2012.

[3] H. E. Hering, "Das wesen des herzalternans," Münchener Med Wochenschr, vol. 4, pp. 1417-1421, 1908.

[4] D. R. Adam, S. Akselrod, and R. J. Cohen, "Estimation of ventricular vulnerability to fibrillation through T-wave time series analysis," Comput Cardiol., vol. 8, pp. 307-310, Sep. 1981.

[5] A. J. Moss, W. Zareba, W. J. Hall, H. Klein, D. J. Wilber, D. S. Cannom, J. P. Daubert, S. L. Higgins, M. W. Brown, and M. L. Andrews, "Prophylactic implantation of a defibrillator in patients with myocardial infarction and reduced ejection fraction," New Engl. J. Med., vol. 346, no. 12, pp. 877-883, 2002

[6] G. H. Bardy, K. L. Lee, D. B. Mark, J. E. Poole, D. L. Packer, R. Boineau, M. Domanski, C. Troutman, J. Anderson, G. Johnson, S. E. McNulty, N. Clapp-Channing, L. D. Davidson-Ray, E. S. Fraulo, D. P. Fishbein, R. M. Luceri, and J. H. Ip, "Amiodarone or an implantable cardioverter-defibrillator for congestive heart failure," New Engl. J. Med., vol. 352 , no. 3 , pp. $225-237,2005$.
[7] D. S. Rosenbaum, L. E. Jackson, J. M. Smith, H. Garan, J. N. Ruskin, and R. J. Cohen, "Electrical alternans and vulnerability to ventricular arrhythmias," N. Engl. J. Med., vol. 330, no. 4, pp. 235-241, 1994.

[8] J. M. Smith, E. A. Clancy, C. R. Valeri, J. N. Ruskin, and R. J. Cohen, "Electrical alternans andcardiac electrical instability," Circulation, vol. 77, no. 1, pp. 110-121, 1988.

[9] B. D. Nearing and R. L. Verrier, "Modified moving average analysis of T-wave alternans to predict ventricular fibrillation with great accuracy," $J$. Appl. Physiol., vol. 92, no. 2, pp. 541-549, 2002.

[10] B. D. Nearing, A. H. Huang, and R. L. Verrier, "Dynamic tracking of cardiac vulnerability by complex demodulation of the T wave," Science, vol. 252, no. 5004, pp. 437-440, 1991.

[11] J. P. Martínez and S. Olmos, "A robust T-wave alternans detector based on the GLRT for the Laplacian noise distribution," in Proc. Comput. Cardiol., pp. 677-680, 2002.

[12] J. P. Martínez and S. Olmos, "Detection of T wave alternans in nonstationary noise: A GLRT approach," in Proc. Comput. Cardiol., 2003. Los Alamitos, CA: IEEE Comput. Soc. Press, pp. 161-164.

[13] R. Goya-Esteban, O. Barquero-Pérez, M. Blanco-Velasco, A. J. Caamaño-Fernández, A. García-Alberola, and J. L. Rojo-Álvarez, "Nonparametric signal processing validation in t-wave alternans detection and estimation," IEEE Trans. Biomed. Eng., vol.61, no. 4, pp. 1328-1338, Apr. 2014.

[14] M. Orini, B. Hanson, V. Monasterio, J. P. Martínez, M. Hayward, P. Taggart, and P. Lambiase, "Comparative evaluation of methodologies for t-wave alternans mapping in electrograms," IEEE Trans. Biomed. Eng., vol. 61 , no. 2, pp. 308-316, Feb. 2014.

[15] S. Nemati, O. Abdala, V. Monasterio, S. Yim-Yeh, A. Malhotra, and G. D. Clifford, "A nonparametric surrogate-based test of significance for t-wave alternans detection," IEEE Trans. Biomed. Eng., vol. 58, no. 5, pp. 1356-1364, May. 2011.

[16] J. P. Martínez and S. Olmos, "Methodological principles of T wave alternans analysis: A unified framework," IEEE Trans. Biomed. Eng., vol. 52, no. 4, pp. 599-613, Apr. 2005.

[17] V. Monasterio and J. P. Martínez, "A multilead approach to t-wave alternans detection combining principal component analysis and the laplacian likelihood ratio method," in Proc. Comput. Cardiol., vol, 34, pp. 5-8, 2007.

[18] V. Monasterio, P. Laguna, and J. P. Martínez, "Multilead analysis of t-wave alternans in the ECG using principal component analysis," IEEE Trans. Biomed. Eng., vol. 56, no. 7, pp. 1880-1890, Jul. 2009.

[19] J. Dezert and F. Smarandache, "An introduction to DSmT," in Advances and Applications of DSmT for Information Fusion, 1st ed., vol. 3, Rehoboth: ARP, 2009, pp. 3-73.

[20] J. Dezert and F. Smarandache(Editors). (2004-2015). Advances and Applications of DSmT for information fusion (Collected works), Vol. 1-4. $\begin{array}{llll}\text { (1st ed.) [Online]. Available: } & \end{array}$ http://www.gallup.unm.edu/ smarandache/DSmT.htm

[21] G. Shafer, "A mathematical theory of evidence," Princeton Univ. Press, 1976.

[22] L. Cholvy, "Using logic to understand relations between DSmT and Dempster-Shafer Theory," in LFA., pp. 321-317, Oct. 2018.

[23] J. Dezert, F. Smarandache, "A new probabilistic transformation of belief mass assignment," in Proc. Int. Conf. on Info Fusion., 2008.

[24] A. Khaustov, S. Neimati, and G. D. Clifford, "An open-source standard T-wave alternans detector for benchmarking," in Proc. Comput. Cardiol., vol. 35, pp. 509-512, Sep. 2008.

[25] Goldberger AL, Amaral LAN, Glass L, Hausdorff JM, Ivanov PCh, Mark RG, Mietus JE, Moody GB, Peng C-K, Stanley HE. PhysioBank, PhysioToolkit, and PhysioNet: Components of a New Research Resource for Complex Physiologic Signals. Circulation 101(23):e215-e220 [Circulation Electronic Pages; http://circ.ahajournals.org/cgi/content/full/101/23/e215]; 2000 (June 13).

[26] J. Pan and W J. Tompkins, "A real-time QRS detection algorithm", IEEE Trans. Biomed. Eng., vol. BME-32, pp. 230-236, 1985.

[27] R. Jane, A. Blasi, J. García, and P. Laguna, "Evaluation of an automatic detector of waveforms limits in holter ECG with the QT database," in Proc. Comput. Cardiol. Los Alamitos, CA: IEEE Computer Society Press, 1997, pp. 295-298.

[28] G. B. Moody, W. E. Muldrow, and R. G. Mark, "A noise stress test for arrhythmia detectors," Comput. Cardiol., vol. 11, pp. 381-384, 1984.

[29] M. Blanco-Velasco, F. Cruz-Roldán, J. I. Godino-Llorente, and K. E. Barner, "Nonlinear trend estimation of the ventricular repolarization segment for t-wave alternans detection," IEEE Trans. Biomed. Eng., vol. 57 , no. 10 , pp. $2402-2412$, Oct. 2010. 